\title{
Modelo de Treinamento para Inserção de Dispositivos Intrauterinos
}

\section{Training Model for Intrauterine Device Insertion}

\author{
Brenda Diniz Rodrigues ${ }^{1 \oplus}$ \\ Camila Fernanda Antunes Castanho Cavaleiro de Macêdo Loureiro \\ Mariseth Carvalho de Andrade \\ Suzana Rodrigues Ramos ${ }^{I}$ \\ Carolina Ribeiro Mainardi \\ Cristina Helena Rama \\ Nara Macedo Botelho ${ }^{I}$
}

\section{PALAVRAS-CHAVE}

- Educação Médica.

- Simulação.

- Dispositivos Intrauterinos.
${ }^{I}$ Universidade do Estado do Pará, Belém, Pará, Brasil.

${ }^{I I}$ Hospital Maternidade Leonor Mendes de Barros, São Paulo, São Paulo, Brasil.
Introdução: Aprender praticando exclusivamente em pacientes é cada vez menos aceitável no ensino médico. O ensino baseado em simulação é uma ferramenta capaz de gerar aquisição de habilidades e competências de forma eficaz e em ambiente controlado, sem causar dano ao paciente. A Ginecologia é uma especialidade médica que depende de treinos bem estruturados, e entre os procedimentos ginecológicos destaca-se a inserção dos dispositivos intrauterinos de cobre. Para aumentar a oferta deste método contraceptivo, é preciso capacitação adequada dos profissionais de saúde. Os simuladores físicos imitam a realidade e auxiliam na aquisição de habilidades e competências, tornando de fundamental importância o ensino baseado em simulação para inserção de dispositivos intrauterinos. Objetivo: Desenvolver um modelo de treinamento para aquisição de habilidades na inserção de dispositivos intrauterinos. Método: Foi desenvolvido um simulador (modelo de utilidade) para inserção de dispositivos intrauterinos, composto por uma base rígida retangular com $17 \mathrm{~cm} \times 15 \mathrm{~cm} \times 21 \mathrm{~cm}$ $(A x L \times P)$, com peso de 420 gramas, confeccionada em acrinolitrilabutadieno estireno (ABS). Acoplada a este suporte, existe uma estrutura semiflexível, composta por um protótipo de canal vaginal, com colo uterino e região vulvar semelhantes à anatomia humana, confeccionada em ácido poliático flexível (PLA-FLEX). Foram selecionados 15 médicos residentes de Ginecologia e Obstetrícia para treinamento teórico-prático com o simulador proposto pelo estudo para capacitação em inserção de dispositivo intrauterino de cobre. O simulador utilizado neste estudo foi avaliado e validado por especialistas em Ginecologia e Obstetrícia. Resultados: Houve melhora estatisticamente significante dos parâmetros avaliados comparando-se a avaliação inicial (pré-treinamento) e final (pós-treinamento) dos residentes, com homogeneidade no desempenho final. Na avaliação inicial, apenas $40 \%$ dos dispositivos ficaram bem posicionados, em contraste com 93,3\% de sucesso de inserção após implementado o modelo de treinamento. Conclusão: Desenvolveu-se um modelo de treinamento baseado em simulação que contribuiu para a aquisição de habilidades de inserção de dispositivo intrauterino de cobre quando aplicado aos residentes de Ginecologia e Obstetrícia. 


\section{KEY-WORDS}

- Medical Education.

- Simulation.

- Intrauterine Devices.

Recebido em: 10/1/19

Aceito em: 1/4/19
ABSTRACT
Introduction: Learning by practicing exclusively on patients is becoming increasingly unacceptable in medical training. Simulation-based teaching is a tool capable of generating skills and competences efficiently and in a controlled environment, without causing harm to patients. Gynecology is a medical specialty that depends on well-structured training; an important gynecological procedure is the insertion of copper intrauterine devices. In order to increase the supply of this contraceptive method to the population the relevant health professionals need to be adequately trained. Physical simulators imitate reality and assist in skills acquisition. Therefore, structured training based on simulation to teach the insertion of intrauterine devices is of fundamental importance to the teaching of gynecology. Objective: To develop a training model for skills acquisition for the insertion of intrauterine devices. Method: A simulator (utility model) was developed for the insertion of intrauterine devices, composed of a rigid rectangular base made of acrylonitrile butadiene styrene, measuring $17 \mathrm{~cm} \times 15 \mathrm{~cm} \times 21 \mathrm{~cm}$ and weighing $420 \mathrm{~g}$. Attached to this support is a semi-flexible structure made of flexible polylactic acid, composed of a prototype vaginal canal, with a uterine cervix and vulvar region, similar to the human anatomy. Fifteen gynecology and obstetrics residents were selected for theoretical and practical training with the simulator proposed by the study for the training of intrauterine copper device insertion. The simulator used in this study was evaluated by specialists in gynecology and obstetrics, who validated it for use in teaching. Results: A statistically significant improvement was found in the evaluated parameters, comparing the initial evaluation and the final evaluation of the residents, whose final performances displayed homogeneity. In the initial evaluation only $40 \%$ of the devices were well positioned, in contrast to a $93.3 \%$ rate of successfull insertion following implementation of the training model. Conclusion: A training model was developed to simulate the performance of copper intrauterine device insertion and contributed to the acquisition of skills for this task when applied to gynecology and obstetrics residents.

\section{INTRODUÇÃO}

Durante anos, o ensino médico foi baseado no modelo proposto inicialmente por Halsted: see one, do one, teach one (veja, faça, ensine), fazendo com que aprender praticando em pacientes se tornasse uma prática ainda presente na atualidade ${ }^{1}$. Com a evolução das metodologias de ensino na área da saúde, surgiram os conceitos habilidades e competências, que trouxeram em conjunto o ensino baseado em simulação ${ }^{2,3}$.

A simulação como forma de aprendizado surgiu nos anos 1930 com o primeiro simulador de voo, Link Trainer. Já o primeiro simulador em medicina foi desenvolvido no início da década de 1960, um boneco para treinamento de respiração boca a boca, que ficou conhecido como Ressuci-Anne ${ }^{4}$. A simulação substitui temporariamente o encontro com o paciente real por modelos artificiais, permitindo que o processo de ensino e aprendizagem ocorra em ambiente seguro, de forma ativa, diminuindo o medo de causar dano durante o aprendizado, com maior retenção de conhecimento, tornando "aprender praticando em pacientes" cada vez menos aceitável ${ }^{4,5,6}$.
A Ginecologia é uma especialidade médica que depende de treinos bem estruturados ${ }^{6}$. Entre os procedimentos ginecológicos, destaca-se a inserção dos dispositivos intrauterinos (DIU) de cobre . O DIU de cobre é um método contraceptivo de longo prazo, pois necessita de substituição somente após dez anos da inserção, é reversível, eficaz, possui poucos efeitos adversos e tem excelente custo-benefício. Em usuárias que fazem revisão médica periódica, as taxas de gravidez são inferiores a 1 em 100 mulheres/ano, mas ainda assim é um método subutilizado no Brasil ${ }^{7,8}$.

A literatura mundial aponta que as habilidades médicas em indicar e inserir corretamente o DIU de cobre estão abaixo do esperado, muitas vezes por imperícia ou por falsas contraindicações, o que reforça a necessidade do desenvolvimento de modelos de treinamento ${ }^{9,10}$.

Para aumentar a oferta deste método contraceptivo à população, é preciso capacitação adequada. O fundamento de modelos de treinamento preconiza que, quanto maior a prática de uma ação, menos complexa ela se torna ${ }^{11}$. Os simuladores 
físicos imitam a realidade, auxiliam o ensino e aprendizado técnico, promovem o ganho de habilidades e competências, e permitem o sucesso final quando a aplicação de um procedimento é direcionada ao paciente ${ }^{1,2,11}$.

\section{OBJETIVO}

Desenvolver um modelo de treinamento para aquisição de habilidades na inserção de dispositivos intrauterinos.

\section{MÉTODO}

Após aprovação do projeto de pesquisa pelo Comitê de Ética e Pesquisa em Seres Humanos (CAAE: 75841617.0.0000.5174), foram selecionados os 15 residentes do primeiro ano do programa de residência médica em Ginecologia e Obstetrícia do Hospital Maternidade Leonor Mendes de Barros (HMLMB), na cidade de São Paulo. Todos assinaram o Termo de Consentimento Livre e Esclarecido. Não houve desistências no decorrer do estudo. Todos os residentes selecionados referiram ausência de treinamento prévio para inserção de DIU de cobre tanto com simulação quanto diretamente em pacientes.

\section{Desenvolvimento do modelo de treinamento}

Trata-se de um simulador físico que configura um modelo de utilidade para inserção de DIU, visando à capacitação teórico-prática na inserção de todos os tipos de DIU em humanos. Os autores depositaram o pedido de patente de modelo de utilidade junto ao Instituto Nacional de Proteção Intelectual (número de processo: BR 202018002915 6).

O modelo de utilidade está representado nas Figuras 1 e 2. A Figura 1 representa uma vista tridimensional do suporte rígido de formato retangular contendo um protótipo anatômico de útero humano hemisseccionado, no interior do qual existe uma cavidade que se assemelha à cavidade uterina normal. Esse suporte rígido tem uma base retangular com $17 \mathrm{~cm}$ no maior ponto de altura, $15 \mathrm{~cm}$ de largura e $21 \mathrm{~cm}$ de profundidade, com peso de 420 gramas. Para o presente estudo, foi confeccionada em acrinolitrilabutadieno estireno (ABS).

Está acoplada a este suporte rígido uma estrutura semiflexível, composta por um protótipo de canal vaginal, colo uterino (com local de engate para pinçamento) e região vulvar semelhantes à anatomia humana. Esta peça foi confeccionada em ácido poliático flexível (PLA-FLEX). A Figura 2 apresenta uma vista tridimensional (frontal e lateral, respectivamente) da peça semiflexível.

O modelo de utilidade foi projetado com o programa Autodesk 3DS Max $2015^{\circ}$, e a confecção foi realizada com impressão tridimensional, utilizando-se a impressora Sethi 3D-S3, com custo final de $\mathrm{R} \$ 487,00$.

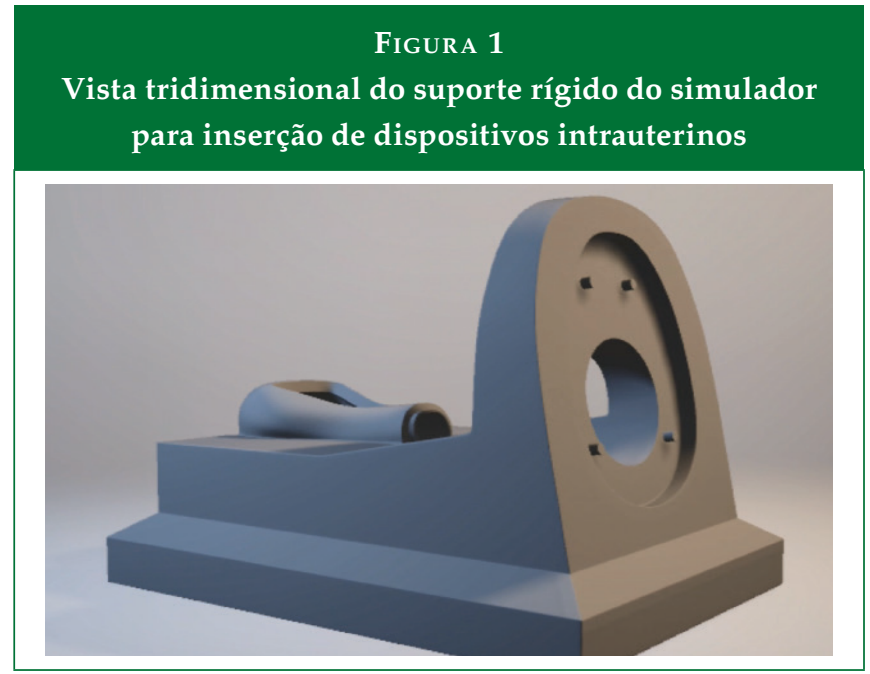

Graças às características do modelo, é possível realizar exame especular, identificação do colo uterino, pinçamento do colo uterino e tração com auxílio de pinça cirúrgica (Pozzi), aferição da histerometria e, em seguida, treinamento da técnica para inserção do DIU de cobre. No estudo foi utilizado o DIU TCU 380-A e foram considerados bem posicionados aqueles de localização fúndica, colocados no centro da cavidade uterina e com os braços do "T de cobre" abertos horizontalmente em direção à topografia dos óstios tubários ${ }^{12}$.

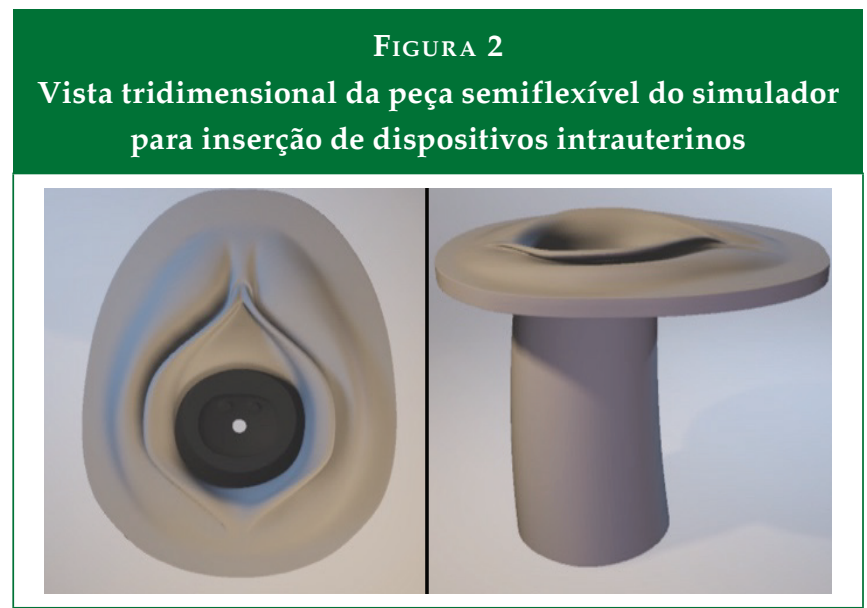

O simulador utilizado no presente estudo foi avaliado por especialistas em Ginecologia e Obstetrícia com auxílio da escala de Likert com cinco pontos. Foi atribuído o conceito excelente ao simulador, caracterizando-o como validado para uso em ensino. 


\section{Treinamento teórico-prático}

O primeiro contato com o simulador visou reconhecer e interagir com o simulador, gerando hipóteses de como deveria ser a técnica correta para inserção do DIU de cobre. Cada residente realizou uma tentativa de inserir o DIU de cobre e foi avaliado segundo quesitos do roteiro com os passos preconizados pelo Ministério da Saúde do Brasil sobre a técnica de inserção do DIU de cobre, transcrito a seguir ${ }^{12}$.

\section{Roteiro para inserção de DIU TCU 380-A}

- Higiene das mãos e paramentação

- Toque vaginal para verificar posição uterina

- Inserção do especulo vaginal

- Antissepsia adequada

- Pinçamento do colo uterino com Pozzi às $12 \mathrm{~h}$

- Histerometria

- Carregar DIU no dispositivo de inserção

- Delimitar histerometria no dispositivo de inserção

- Introdução do dispositivo de inserção

- Liberação dos braços do DIU

- Certificar o posicionamento do DIU (fúndico)

- Retirada do dispositivo de inserção

- Secção dos fios do DIU $(2-3 \mathrm{~cm})$

- Retirar pinça Pozzi e verificar a hemostasia

- Retirar espéculo vaginal

- DIU bem posicionado?

Após o primeiro contato com o simulador, foram ministradas aulas conceituais com recursos audiovisuais que abordavam introdução geral sobre o DIU de cobre e seu mecanismo de ação, eficácia, tempo de duração, vantagens, desvantagens, critérios de elegibilidade, efeitos colaterais, complicações e seus manejos.

Por fim, foi descrita a técnica adequada para inserção do DIU TCU 380-A, com elaboração de um roteiro. O instrutor inicialmente demonstrou a técnica de inserção do dispositivo intrauterino com o auxílio de um vídeo demonstrativo e em seguida com demonstração prática no simulador físico.

Passados 15 dias da exposição teórico-prática, cada aluno participante da pesquisa teve um período de treinamento livre controlado em posse do roteiro, com 30 minutos de prática no simulador. Nessa etapa, foi fornecido também um feedback quanto a erros e acertos anotados na primeira avaliação.

Após 15 dias do treino livre controlado, cada aluno, separadamente, simulou a inserção do DIU de cobre no modelo de utilidade, fazendo nesse momento a narrativa de cada passo, que foi avaliado pelo instrutor com auxílio do roteiro para colocação de DIU do protocolo de pesquisa.

\section{Avaliação estatística}

A amostra foi estabelecida por conveniência. Os dados da pesquisa foram armazenados em planilhas do Microsoft Excel $2016^{\circ}$ e submetidos a análise estatística no programa Bioestat $5.3^{\odot}$. Foi utilizado o teste de normalidade de Shapiro-Wilk, o teste $\mathrm{t}$ de Student pareado e o teste G Aderência. Foram considerados significantes resultados com $p<0,05$.

\section{RESULTADOS}

Participaram do estudo 15 alunos e não houve desistência. Quanto à média de acertos, foi observada melhora estatisticamente significante ao término do treinamento proposto em relação à avaliação inicial dos residentes (Figura 3). O diagrama de extremos e quartis (Figura 4) demonstra que durante a avaliação inicial havia grande dispersão do conhecimento, em contraste com a homogeneidade do desempenho final.

No primeiro contato com o simulador, a média de acertos dos residentes foi de 59,2\% por questão, e a média geral de acertos por aluno foi de $63,3 \%$. Já na avaliação final, os resultados obtidos foram 92,1\% e 98\%, respectivamente (Tabela 1).

Na avaliação inicial, apenas $40 \%$ dos dispositivos ficaram bem posicionados, em contraste com 93,3\% de sucesso de inserção após implementado o modelo de treinamento (Figura 5).

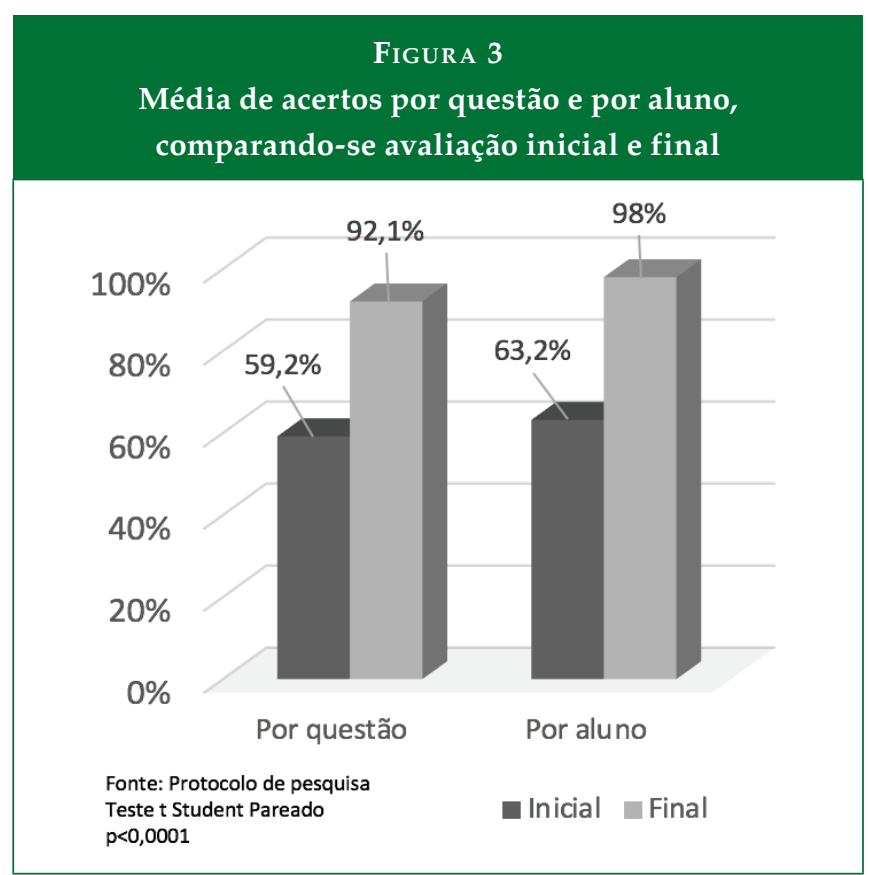


Figura 4

Diagrama de extremos e quartis

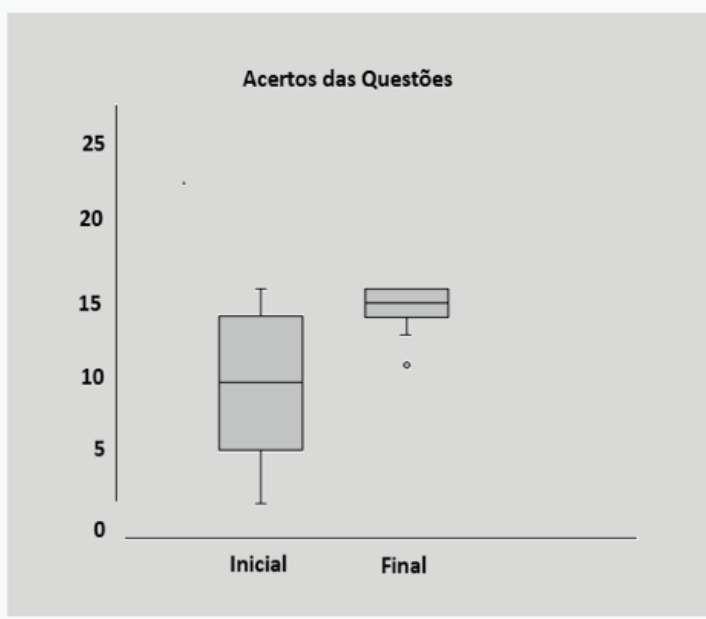

Fonte: Protocolo de Pesquisa

Teste $\mathrm{t}$ Student pareado

Valor do $P<0,0001$
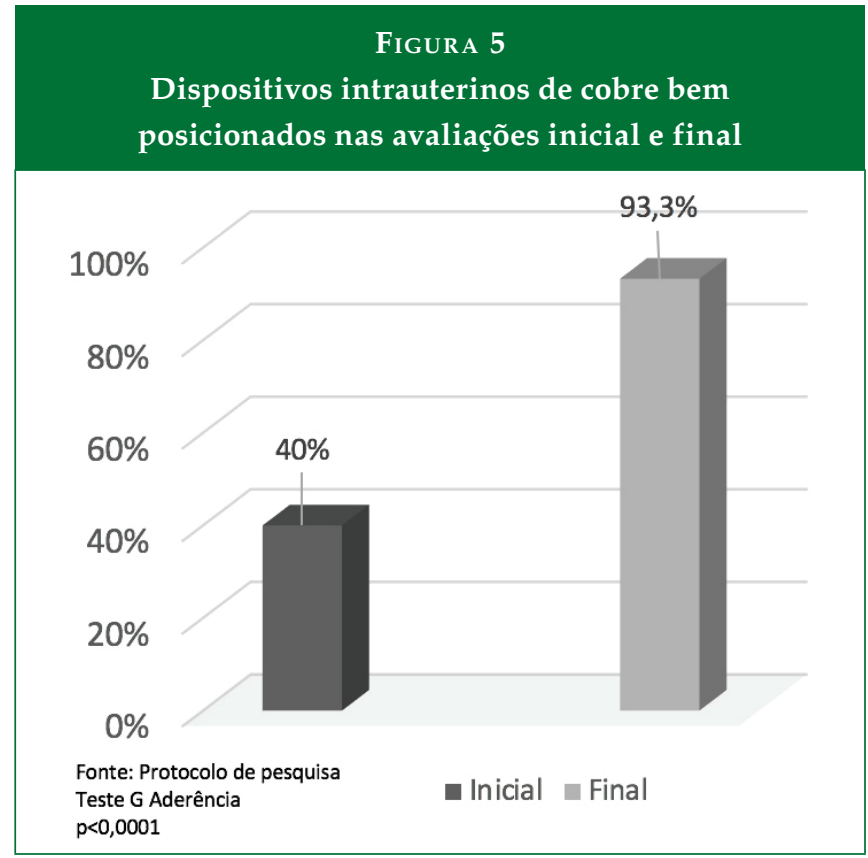

\begin{tabular}{|c|c|c|c|c|c|}
\hline \multicolumn{6}{|c|}{ TABELA 1} \\
\hline Questões da avaliação & \multicolumn{2}{|c|}{ Avaliação inicial } & \multicolumn{2}{|c|}{ Avaliação final } & \multirow{2}{*}{$\begin{array}{l}\mathrm{p} \text { - valor } \\
<0,0001^{*}\end{array}$} \\
\hline 1. Higiene das mãos e paramentação adequada & 1 & $6,7 \%$ & 14 & $93,3 \%$ & \\
\hline 2. Exame físico adequado & 6 & $40,0 \%$ & 10 & $66,7 \%$ & $0,0465^{*}$ \\
\hline 3. Inserção apropriada do espéculo vaginal & 15 & $100,0 \%$ & 15 & $100,0 \%$ & - \\
\hline 4. Fez antissepsia adequada & 7 & $46,7 \%$ & 12 & $80,0 \%$ & $0,0164^{*}$ \\
\hline 5. Pinçamento do colo uterino & 15 & $100,0 \%$ & 15 & $100,0 \%$ & - \\
\hline 6. Histerometria adequada & 14 & $93,3 \%$ & 15 & $100,0 \%$ & 0,6291 \\
\hline 7. Carregou o DIU corretamente no dispositivo de inserção & 14 & $93,3 \%$ & 15 & $100,0 \%$ & 0,6291 \\
\hline 8. Delimitou histerometria dispositivo de inserção & 6 & $40,0 \%$ & 14 & $93,0 \%$ & $<0,0001^{*}$ \\
\hline 9. Introdução adequada do dispositivo de inserção & 9 & $60,0 \%$ & 13 & $86,7 \%$ & $0,0431^{*}$ \\
\hline 10. Liberou braço do DIU corretamente & 7 & $46,7 \%$ & 14 & $93,3 \%$ & $<0,0001^{*}$ \\
\hline 11. Certificou posicionamento do DIU & 4 & $26,7 \%$ & 13 & $86,7 \%$ & $<0,0001^{*}$ \\
\hline 12. Retirada do dispositivo de inserção & 8 & $53,3 \%$ & 14 & $93,3 \%$ & $0,0008^{*}$ \\
\hline 13. Secção do fio apropriada $(2-3 \mathrm{~cm})$ & 7 & $46,7 \%$ & 15 & $100,0 \%$ & $<0,0001^{*}$ \\
\hline 14. Retirou pinça do colo e hemostasia adequada & 8 & $53,3 \%$ & 13 & $86,7 \%$ & $0,0107^{*}$ \\
\hline 15. Retirou espéculo vaginal corretamente & 15 & $100,0 \%$ & 15 & $100,0 \%$ & - \\
\hline 16. Posicionamento adequado do DIU & 6 & $40,0 \%$ & 14 & $93,3 \%$ & $<0.0001^{*}$ \\
\hline Média geral de acertos por questão & 8,9 & $59,2 \%$ & 13,8 & $92,1 \%$ & - \\
\hline Média geral de acertos por aluno & 9,5 & $63,3 \%$ & 14,7 & $98,0 \%$ & - \\
\hline
\end{tabular}

* Teste G Aderência. 


\section{DISCUSSÃO}

Houve melhora progressiva estatisticamente comprovada na média de acertos, tanto por questão quanto por aluno, após o treinamento com simulação, corroborando resultados da literatura sobre ensino baseado em simulação. Bartz et al. ${ }^{11}$ descrevem um aumento de conhecimento sobre métodos contraceptivos intrauterinos com auxílio de simulação prática no consultório de Ginecologia e Obstetrícia, gerando relações mais favoráveis quanto ao método ${ }^{11}$.

O modelo de treinamento baseado em simulação proposto por Oti et al..$^{13}$ gerou importante ganho de habilidades de atividades laparoscópicas, em concordância com a literatura, que evidencia a redução do nível de dificuldade técnica após treinos em simuladores de alta ou baixa fidelidade ${ }^{14,15}$.

Os autores do presente estudo destacam a importância do feedback durante o treinamento como ferramenta de construção do conhecimento, estimulando o pensamento crítico dos alunos acerca dos erros cometidos na avaliação inicial, o que possibilitou um conhecimento mais homogêneo ${ }^{16}$.

Publicações diversas descrevem os benefícios do ensino baseado em simulação e da possibilidade de aprender com erro em ambientes controlados com simulação ${ }^{14,16,17}$. A reflexão sobre o erro é um dos pontos principais na construção do conhecimento, pois se quantifica o desempenho de aprendizagem, gerando impacto de forma virtuosa na vida real ${ }^{18}$.

No Brasil e no mundo, a falta de conhecimento técnico sobre o DIU de cobre leva este método altamente eficaz a ser subutilizado ${ }^{19,20}$. É preciso desenvolver modelos de treinamento acessíveis para simulação de contracepção intrauterina a fim de modificar essa situação, contribuindo para aumentar o número de indicações do método por profissionais capaci$\operatorname{tados}^{21,22}$.

\section{CONCLUSÃO}

Desenvolveu-se um modelo para treinamento baseado em simulação que contribuiu para a aquisição de habilidades na inserção de dispositivo intrauterino de cobre quando aplicado a residentes de Ginecologia e Obstetrícia.

\section{REFERÊNCIAS}

1. Torkington J, Smith SG, Rees BI, Darzi A. The role of simulation in surgical training. Ann R Coll Surg Engl. 2000; 82: 88-94.

2. Miller GE. The assessment of clinical skills/competence/ performance. Acad Med. 1990; 65: 63-7.

3. Aggarwal R, Darzi A. Simulation to enhance patient safety: why aren't we there yet? Chest. 2011; 140(4):854-58.
4. Cooper JB, Taqueti VR. A brief history of the development of manequin simulators for clinical education and training. PostgradMed J. 2008; 84(997):563-70.

5. Dourado ASS, GiannellaI TR. Ensino baseado em simulação na formação continuada de médicos: análise das percepções de alunos e professores de um Hospital do Rio de Janeiro. RevBrasEduc Méd. 2014; 38 (4): 460-69

6. Flato UAP, Guimarães HP. Educação baseada em simulação em medicina de urgência e emergência: a arte imita a vida. Rev Bras Educ Méd. 2011; 9(5):360-64.

7. Giordano MV, Giordano LA, Panisset KS. Dispositivo intrauterino de cobre. Femina. 2015; 43 (1): 15-20.

8. Schubert FD, Herbitter C, Fletcher J, Gold M. IUD Knowledge and Experience Among Family Medicine Residents. Fam Med. 2015;47(6):474-77.

9. Dermish AI, Turok DK, Jacobson JC, Murphy PA, Saltzman $\mathrm{HM}$, Sanders. Evaluation of an intervention designed to improve the management of difficult IUD insertions by advanced practice clinicians. Contraception. 2016; 93 (6): 533-38.

10. Dermish AI, Turok DK, Jacobson JC, Flores MES, McFadden M, Burke K. Failed IUD insertions in community practice: an under-recognized problem? Contraception. 2013;87(2):182-86.

11. Bartz D, Paris A, Maurer R, Gardner R, Johnson N. Medical student simulation training in intrauterine contraception insertion and removal: an intervention to improve comfort, skill, and atitudes. Contraception. 2016; 1:3

12. Brasil. Ministério da Saúde. Secretaria de Atenção à Saúde. Departamento de Atenção Básica. Saúde sexual e saúde reprodutiva / Ministério da Saúde, Secretaria de Atenção à Saúde, Departamento de Atenção Básica. Brasília: Ministério da Saúde. 2010. p.191-205.

13. Oti AT et al. Developmentof a laparoscopic training modelusing a smartphone. RevColBrasCir 2017; 44(5): 471-75.

14. Bouet PE et al.Training of medical students for pelvic examination: Benefits of teaching on anatomic models. J Gynecol Obstet Biol Reprod. 2016;45(7):679-84

15. Hugon-Rodin J et al. Learning the gynecological examination on low-fidelity simulation: Impact on the feelings of medical students. Gynecol Obstet Fertil Senol. 2017;45(5):291-98.

16. Varga CRR et al. Relato de experiência: o uso de simulações no processo de ensino-aprendizagem em medicina. Rev Bras Educ Méd. 2009; 33(2):291-97.

17. Timms C, Speirs K. Student perspectives on post-simulation debriefing. Clin Teach 2015;12(6):418-22.

18. Nystrom S, Dahlberg J, Edelbring S, Hult H, Dahlgren MA. Debriefing practices in interprofessional simulation with 
students: a sociomaterial perspective. BMC Med Educ. 2016; 16:148-55.

19. D'Arcangues C. Worldwide use of intrauterine devices for contraception. Contraception. 2007;75(6 Suppl):2-7.

20. Thompson KM, Stern L, Gelt M, Speidel JJ, Harper CC. Counseling for IUDs and implants: are health educators and clinicians on the same page? Perspect Sex Reprod Health. 2013;45(4):191-5.

21. Lichowski AT, Anderson BL, Power ML, Raglan GB, Espey E, Schulkin J. Obstetrician-gynecologists and contraception: long-acting reversible contraception practices and education. Contraception. 2014;89(6):578-83.

22. Gomez AM, Hartofelis EC, Finlayson S, Clark JB.Do Knowledge and Attitudes Regarding Intrauterine Devices Predict Interest in Their Use? Womens Health Issues. 2015;25(4):359-65

\section{CONTRIBUIÇÃO DOS AUTORES}

Todos os autores participaram da concepção do trabalho, análise de dados, revisão bibliográfica e redação do manuscrito, aprovando a versão final.

\section{CONFLITO DE INTERESSES}

Os autores declaram que não possuem qualquer conflito de interesses.

\section{ENDEREÇO PARA CORRESPONDÊNCIA}

Brenda Diniz Rodrigues

Laboratório de Cirurgia Experimental da Universidade do Estado do Pará

Tv. Perebebuí, 2623 - Marco, Belém - PA, CEP: 66087-662

E-mail: brendadinizrodrigues@gmail.com 